\title{
ELLIS-VAN CREVELD SYNDROME IN IDENTICAL TWINS*
}

\author{
BY
DANIEL GOOR, YAACOV ROTEM, ABRAHAM FRIEDMAN, AND HENRY N. NEUFELD

From the Heart Institute and Department of Pediatrics of the Tel-Hashomer Government Hospital in Israel

Received January 6, 1965

In 1933 McIntosh (Holt and McIntosh, 1933) described abnormal findings in a girl who had hereditary ectodermal dysplasia, associated with polydactylism and chondrodystrophy. Ellis and van Creveld (1940) described two additional cases, thereby concluding that this was a new syndrome with which their names have since been eponymously associated.

Since then reports on the subject have grown considerably (Gatto, 1951; Weller, 1951; Keizer and Schilder, 1951; Caffey, 1952; MacGregor, 1954; Metrakos and Fraser, 1954; Medulla, 1954; Weiss and Crosett, 1955; Chauss, 1955; Debré et al., 1955; Turner, 1956;.Uehlinger, 1957; Darling, Rothney, and Craig, 1957; Smith and Hand, 1958; Mitchell and Waddell, 1958; Walls, Altman, and Winslow, 1959; Douglas, Schonholtz, and Geppert, 1959; Alvarez-Borja, 1960; Husson and Parkman, 1961; Ferrero, Pozo, and Morresi, 1961; Ellis and Andrew, 1962; Kushnick, Paya, and Mamunes, 1962; Giknis, 1963; Behar and Rachmilewitz, 1964; and others). The syndrome consists of chondrodystrophy, ectodermal dysplasia, polydactyly, and congenital malformation of the heart. It becomes apparent that the syndrome may be incomplete and varying degrees of each malformation may exist.

Although a congenital malformation of the heart is a part of the syndrome, cases without this have been reported (Caffey, 1952; Metrakos and Fraser, 1954; Mitchell and Waddell, 1958; AlvarezBorja, 1960). Often, however, when heart disease was present, precise diagnosis was not established.

Mitchell and Waddell (1958) were the first to report a pair of sibs who had been completely studied. Metrakos and Fraser (1954) described two cases in sibs and two in dizygotic twin brothers. Another pair of sibs with "incomplete" Ellis-van Creveld syndrome was recently reported by Alvarez-Borja (1960).

This report concerns two identical male twins with the syndrome and with the cardiac anomalies. Special emphasis will be given to the high incidence of persistent common atrio-ventricular canal as a part of the syndrome.

\section{CASE REPORTS}

Identical twins (J.M. and J.S.), aged 17 years, were born to parents who were first cousins after a normal pregnancy and without any complications at birth. The family tree contains several instances of cousin marriages over past generations. The family is Jewish, of Iraqi origin. Five brothers and sisters and the parents are of dark complexion with black eyes and hair, while the twins themselves are blond with blue eyes. Five brothers of the mother are tall with black eyes and hair while the sixth is blond with blue eyes and is $160 \mathrm{~cm}$. in height.

* Supported in part by Grant H-6057 from the National Heart Institute, National Institutes of Health, Bethesda, Md., U.S.A. 


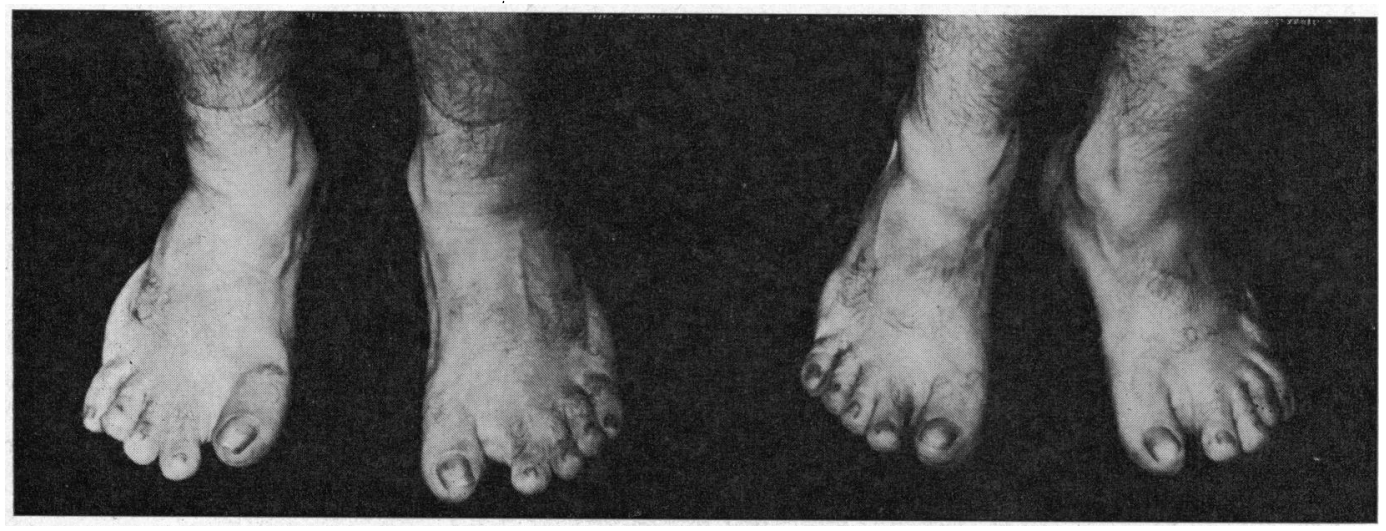

FIG. 1.-Feet in both twins. Left (J.S.), right (J.M.). The sixth post-axial digits have been amputated. The position of the toes appears abnormal, whereas the nails appear normal.

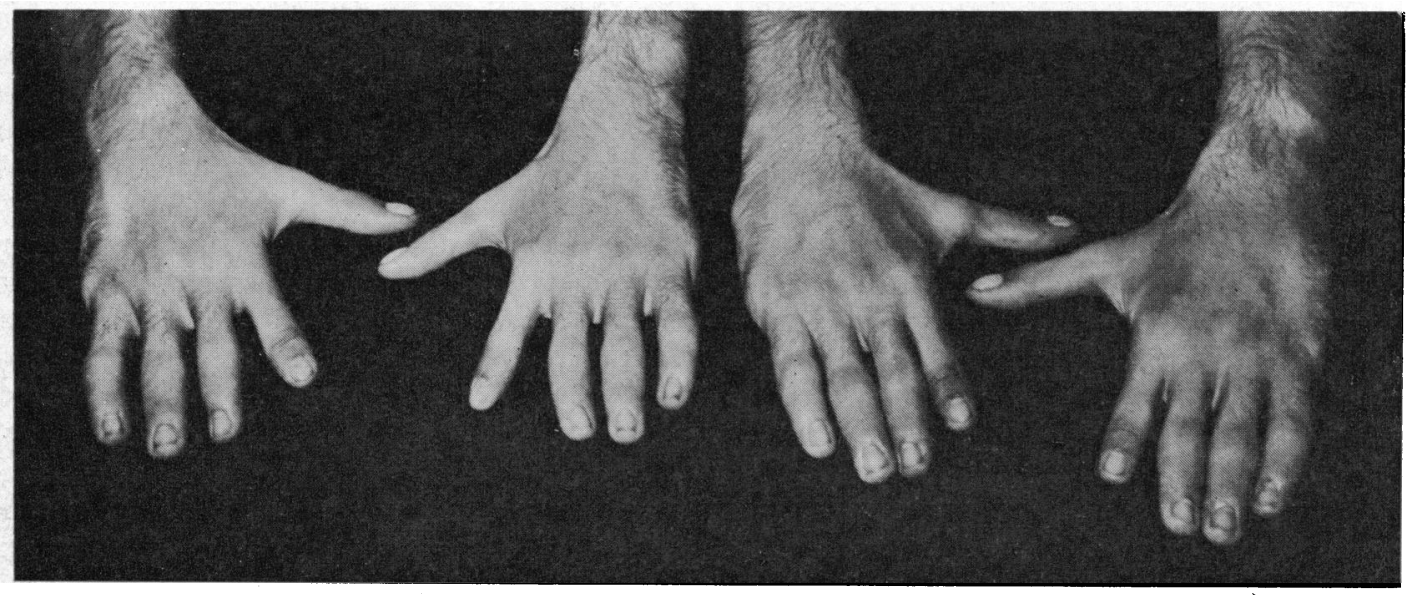

Fig. 2.-Left (J.S.) and right (J.M.). The sixth post-axial fingers have been removed. The fingers appear bizarre. The nails are normal.

After birth, sixth post-axial digits on hands and feet were excised in both twins. They were regarded as healthy until the age of 10 when a cardiac murmur was discovered on routine examination.

The clinical diagnosis of Ellis-van Creveld syndrome was first made in 1958 when they were 12 years old, at which time it was first noticed that their limbs were short relative to the trunk in comparison with other children. They were referred to the hospital in 1963 for full cardiac evaluation.

Examination. The brothers were 17 years old with identical normal and abnormal findings. They appeared to be of normal intelligence and had a particularly well-developed sense of humour. Their lower limbs were relatively short and both had genu valgum. Their genital organs were normally developed. Their height was $158 \mathrm{~cm}$. (upper segment $85 \mathrm{~cm}$., lower segment $73 \mathrm{~cm}$.), span $154 \mathrm{~cm}$., weight $50 \mathrm{~kg}$.: the shape of the head was normal with slight prognathism. They had fair hair and normal nails (Fig. 1, 2). The skin appeared less elastic than usual and they both had pectus excavatum. The limbus of the cornea was not perfectly rounded and resembled more a drope. Myopia was present in the left eye and astigmatism in the right eye. Teeth were normal and the hard palate had a normal shape and posterior border. There was no cyanosis. The neck veins appeared slightly prominent but there were no signs of peripheral congestive heart failure. The peripheral pulses were normally palpable.

Examination of the heart in both showed active but regular heart action. The apex was palpable at the sixth intercostal space at the anterior axillary line. No thrills were palpable. In both an ejection type 

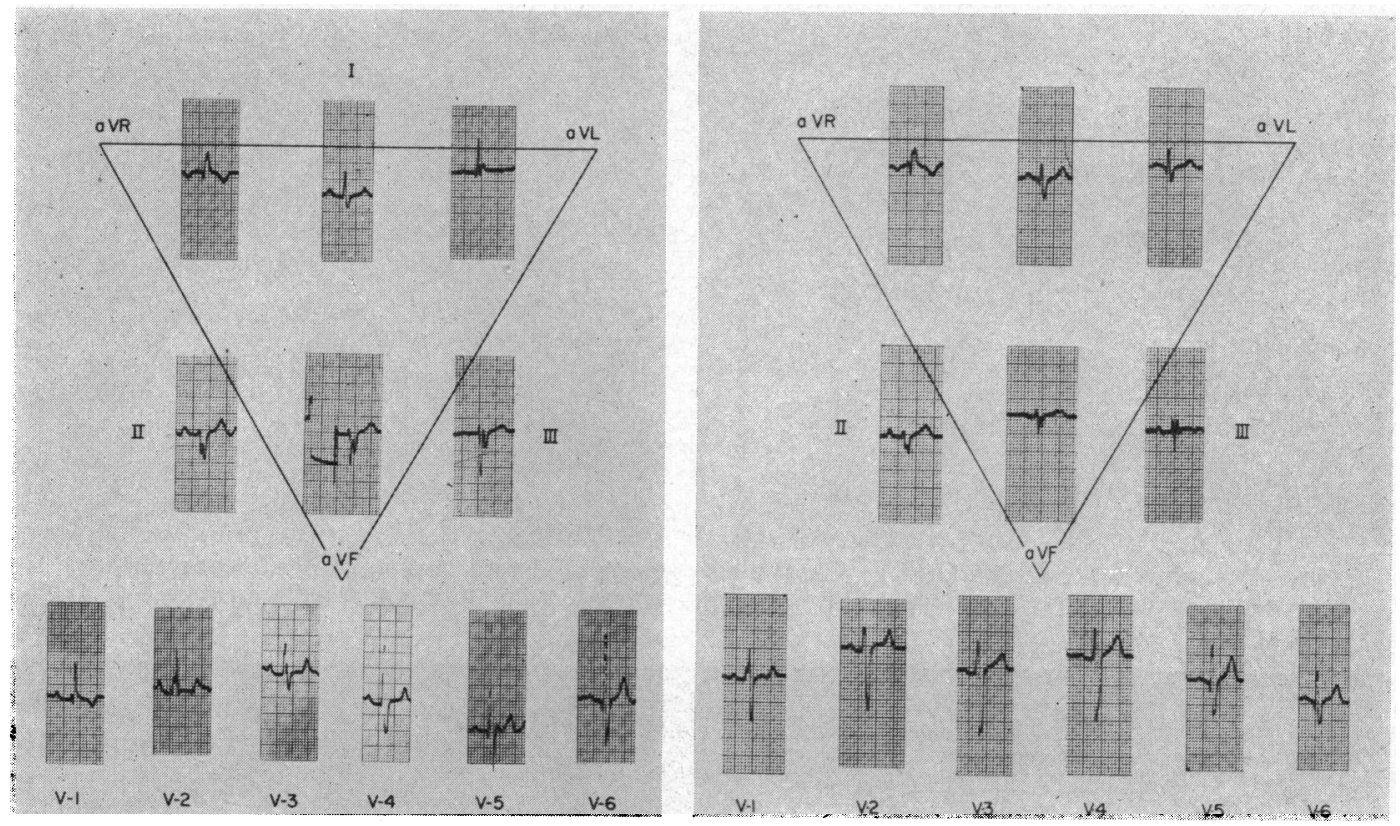

FIG. 3.-Left (J.S.) and right (J.M.). Electrocardiograms showing similar features in both cases. Normal sinus rhythm. The mean manifest electrical axis of the QRS complex is $-80^{\circ}$ with the vectoral forces directed counterclockwise in the frontal plane. The main mass of the frontal vector was above the zero line. An rSR1 pattern in lead V1. Signs of combined ventricular hypertrophy.

systolic murmur was audible, grade 3/6 (J.M.) and grade 2/6 (J.S.). The second sound at the pulmonary area was accentuated and split, and the splitting did not change with respiration. In addition, a short harsh diastolic murmur was heard over the lower sternal area, being somewhat louder in one of the twins (J.S.).

The cardiogram (Fig. 3) showed normal sinus rhythm. The mean manifest electrical axis of the QRS complexes was about -80 degrees. The vector in the frontal plane was directed counterclockwise with its main mass above the zero line. The horizontal plane showed signs of right bundle-branch block. Over the left chest a $q R$ pattern was present with relatively tall $R$ waves.

Radiographic examination showed moderate cardiomegaly with a prominent pulmonary trunk and increased pulmonary arterial vasculature (Fig. 4).

Right heart catheterization in both twins revealed a large left-to-right shunt at the atrial level. In patient J.S. the catheter was introduced via the right arm, but in J.M. it was introduced via the left arm and a persistent left superior vena cava emptying into the coronary sinus was found. The pressure in the right atrium was normal in both patients. The right ventricle and pulmonary arteries were entered in both and the pressures recorded were moderately raised. No pressure gradient was present between the right ventricle and pulmonary trunk (J.M., right ventricular pressure $37 / 0-5$, pulmonary arterial pressure $37 / 12 \mathrm{~mm}$. $\mathrm{Hg}$; J.S., right ventricular pressure 45/0-5, pulmonary arterial pressure $45 / 15 \mathrm{~mm}$. $\mathrm{Hg}$ ).

The lower limbs were shorter than usual with genu valgum, particularly in the right leg (both children) and a tendency to pes cavus. Their legs appeared to be proportionately shorter than their hands. Operative scars were found in the region of the amputation of the supernumerary digits (post-axial in both hands and in both legs) (Fig. 1, 2). The remaining fingers had a bizarre appearance, resembling sausages, the phalanges being out of proportion to the fingers. The fingers showed considerably diminished mobility. The palms showed a four-finger line (Fig. 5). The skin ridges on the fingers were unusual. The fingerprint of the index was normal with the configuration of loops and radial triradius.

The radiological findings in the limbs were also identical in both twins and were most prominent in the tubular bones. The femur was shorter than normal. The tibia was also shorter with depression of the lateral condyle. The fibula was shorter in its upper portion. The feet showed short metatarses, particularly 


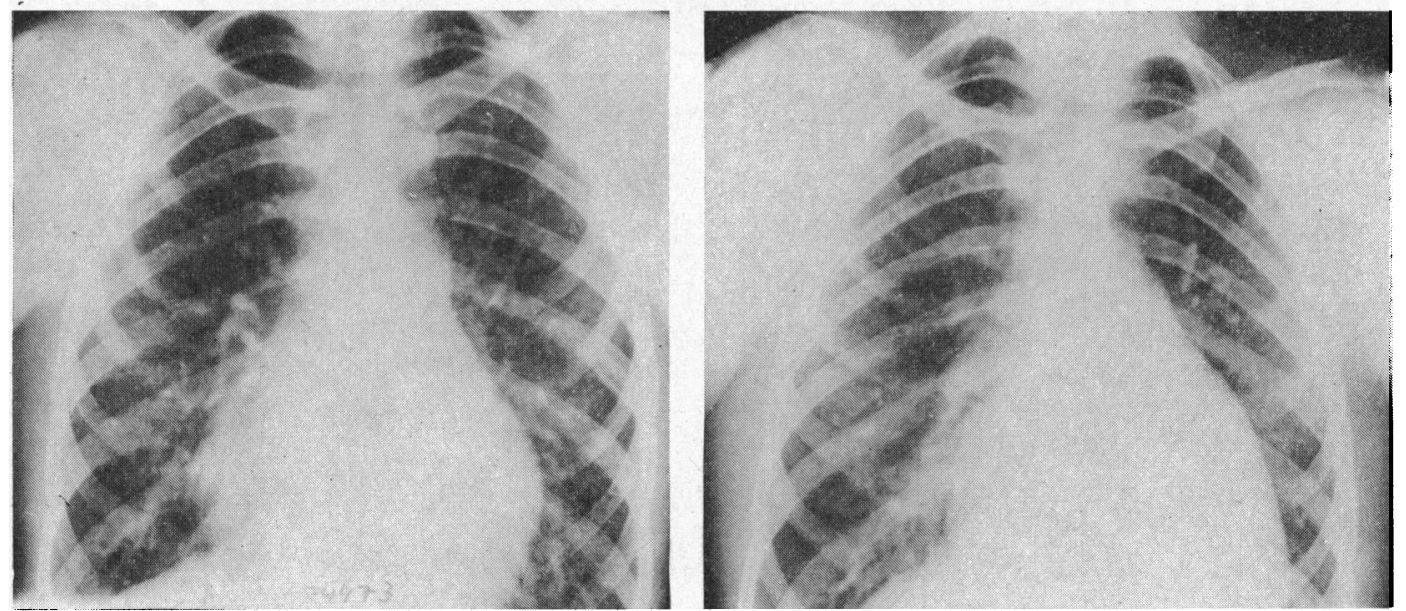

FIG. 4. - Left (J.S.) and right (J.M.). Chest films showing moderate cardiomegaly with prominence of the main pulmonary trunk. The pulmonary arterial vasculature is markedly increased. 

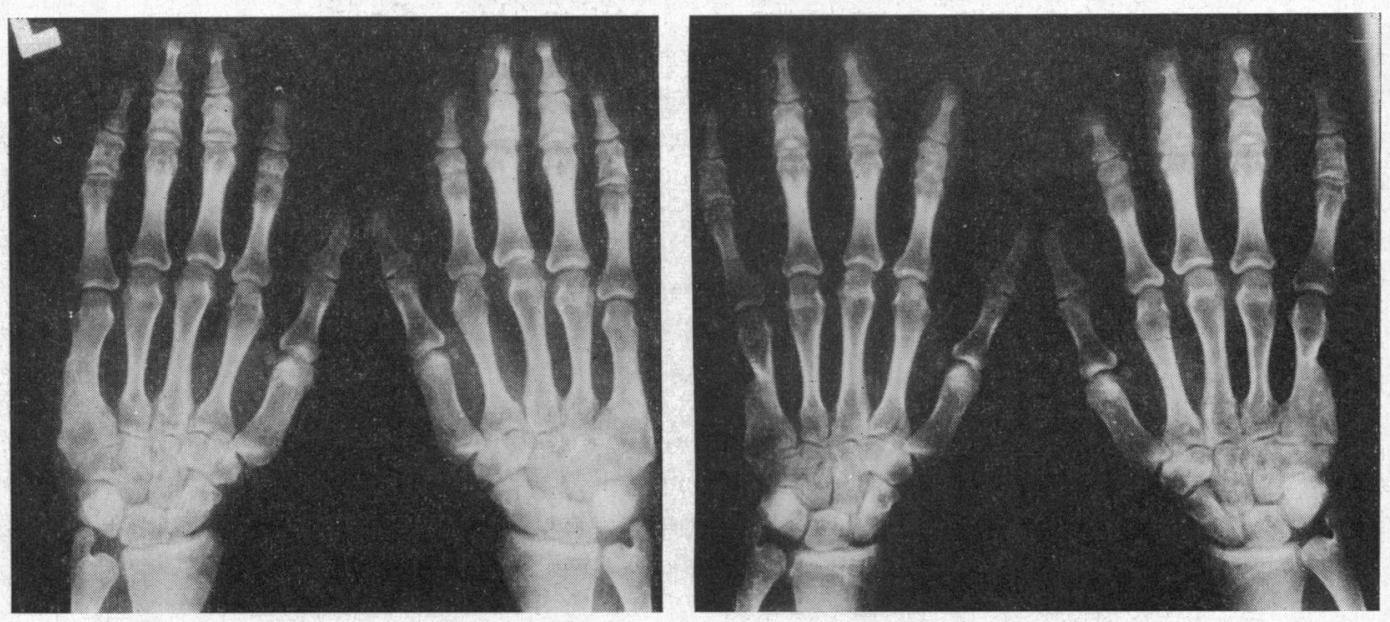

FIG. 7.-Left (J.S.) and right (J.M.). The middle phalanges show much shortening while the metacarpal bones are only slightly shortened; malformation of the distal row of the carpal bones. Note the synostosis of the proximal and middle phalanges of the second finger in both hands. The fifth metacarpal bone is deformed at the place where the accessory finger was amputated.

the first ones, and the middle phalanges were shorter than normal (the proximal and distal ones were normal) (Fig. 6).

Their hands showed a similar picture with shortening of the metacarpals and of the middle phalanges. In both hands there was synostosis of the fifth and the sixth metacarpals and of the middle and distal phalanges of the second finger (Fig. 7).

\section{Discussion}

The Ellis-van Creveld syndrome is a hereditary disease with recessive transmission (McKusick, 1959). It has been described in families with consanguinity (Metrakos and Fraser, 1954; Mitchell and Waddell, 1958), in brothers (Keizer and Schilder, 1951; Debré et al., 1955; Alvarez-Borja, 1960), and in one case of dizygotic twins (Metrakos and Fraser, 1954). Chromosomal examinations have until now been normal.

This report concerns identical twins without any familial disease in the previous two generations, as the parents could not recall any cases of polydactyly or heart disease in the family. The only other example of twins with Ellis-van Creveld has been described by Metrakos and Fraser (1954), and there were considerable differences in both the pathological and the normal findings in the two boys. One of them had microcephaly and mental retardation, cataracts in both eyes, and absence of lower gingival sulcus, and these were not found in the other; the chondrodystrophy and polydactyly, as well as a normal heart, were present in both.

It is of interest that in the twins described here, as well as in the heterozygotic twins described by Metrakos and Fraser (1954), the pathological findings of the four major features typical of the Ellisvan Creveld syndrome when present were identical in both twins. The twins described by them differ from others mainly in that they were heterozygous and did not have a congenital heart lesion.

Of the pathological findings in the Ellis-van Creveld syndrome, chondrodystrophy is the most common. It affects only the tubular bones and, therefore, the patients have short stature with short extremities. Radiologically, apart from the usual features of chondrodystrophy, there is an apparent tendency for classical findings in certain sites (Ellis and Andrew, 1962).

(1) The more distal bones are more severely affected so that the terminal digits show the more severe changes.

(2) The epiphysis of the tibia has the appearance of a wedge with the head medially and the apex laterally. 
(3) Shortening of the fibula limited to the distal portion (thus differentiating it from achondroplasia).

In our patients, the clinical chondrodystrophy was discovered at the age of 12 years. This late detection is unusual and indicates that the changes were not severe. The radiological examination showed the most affected bones to be the middle phalanges (and not the distal ones): the metacarpal bones and humeri, the metatarsal bones, tibiae, fibulæ, and the femora were also shortened (Fig. 6, 7). The epiphysis of the tibia was normally preserved apart from a slight depression of a lateral condyle, and the lateral condyle of the femur appeared slightly deformed.

It appears that although, typically, chondrodystrophic changes are localized in the tubular bones the exact location of the pathological changes may differ. In our patients the pathological findings were mainly in the humeri and in the middle phalanges, whereas the epiphysis of the tibia and the distal end of the fibula, as well as the terminal phalanges, did not show much change. It could be that the appearance of the classical radiographic features depends upon the severity of the disease or upon the age. Their being older than most cases described may account for the somewhat different radiological picture of our patients.

Another major manifestation of Ellis-van Creveld syndrome is polydactyly. It may appear sporadically as a familial feature, or it may be a part of other syndromes. Another possibility is its appearance in association with other abnormalities, for instance congenital heart block (Lynch and Engle, 1961).

The polydactyly is variable. Most of the cases had 6 fingers on both hands but there were some with the normal number (Keizer and Schilder, 1951; Debré et al., 1955). Seven fingers on both hands have also been noted (Weller, 1951). Most did not show polydactyly of the feet (Ellis and van Creveld, 1940; MacGregor, 1954; Metrakos and Fraser, 1954; Weiss and Crosett, 1955; Chauss, 1955; Walls et al., 1959; Husson and Parkman, 1961; Ellis and Andrew, 1962; and others), but in some six toes were also noted (Caffey, 1952; Turner, 1956) as well as in our two cases. If the complete syndrome includes polydactyly of the hands and the feet then anything less than this would represent a forme fruste of the disease. That being so, it becomes possible that polydactyly could be entirely absent in Ellis-van Creveld and in some members of a family described by Debré et al. (1955) it was indeed absent.

Another important pathological finding in Ellis-van Creveld is the congenital heart defect. There are two points of special interest in the patients described. The presence of the same congenital heart defect in twins and the presence of a form of persistent common atrio-ventricular canal.

The presence of the congenital heart disease in twins justifies speculation concerning the genetic origin when the twins are identical. Some salient points are worth reiterating. From the review by Ross (1959) of 37 pairs of twins with congenital heart disease, 11 were monozygotic and 26 were dizygotic twins. In 2 pairs of the 11 monozygotic twins, both had a congenital heart lesion, while in the remaining 9 only one twin was affected. In the first of the pairs both brothers had patent ductus arteriosus and in the second pair one had a ventricular septal defect and the other an atrial septal defect. Of the 26 dizygotic twins only two had a cardiac lesion in both of a pair: the diagnoses were not mentioned.

Persistent common atrio-ventricular canal is rare. Among 357 cases of congenital heart disease proven at necropsy and described by Fontana and Edwards (1962), only 15 were found to have this anomaly. The presence of a rare form of congenital heart disease in both the identical twins strongly favours a genetic ætiology.

Congenital heart lesions in reported cases of Ellis-van Creveld approach a 60 per cent incidence (Ellis and Andrew, 1962; Giknis, 1963): in one review (Giknis, 1963) 20 of 36 Ellis-van Creveld patients had heart lesions. From these 20 patients the hearts were examined at necropsy only in 11 , of whom 7 were found to have a single atrium, 1 an ostium primum defect, 2 a ventricular septal defect, and 1 an intact septum. In an additional case report (Behar and Rachmilewitz, 1964) of a patient who came to necropsy at the age of 32 a single atrium was also found.

It is difficult to review all the clinical cardiological diagnoses since most of the cases were described 
without special investigations and before the full criteria of persistent common atrio-ventricular canal had been established: the presence of shunt, with or without mitral regurgitation had a classical electrocardiographic picture consisting of a typical vector loop on the frontal plane. Nevertheless, there is a clinical report of a case (Ellis and Andrew, 1962) where atrial septal defect of the ostium primum type was established.

The presence of single atrium in 9 of the 12 cases coming to necropsy is very high and, if it be taken into account that the ventricular septal defect cases also may have been of an atrio-ventricular canal type, then the frequency of this type of lesion becomes even greater.

The electrocardiographic picture is similar in the various forms and degrees of common atrioventricular canal or so-called endocardial cushion defects. Neufeld et al. (1961) have explained the changes in this lesion as possibly resulting from displacement of the conduction tissue. Examples were presented from the different forms and degrees of this condition from a single cleft of one valve to the full form of complete common canal, showing that the cardiographic feature common to all was the characteristic vector loop in the frontal plane (Toscano-Barbosa, Brandenburg, and Burchell, 1956). It should be noted that in cases of single atrium the morphological situation is somewhat similar to that of common atrio-ventricular canal, and the embryological deviation may be similar for all these.

In both of our patients the diagnosis of a common atrio-ventricular canal with a large left-toright shunt at atrial level was made. Recently another patient with Ellis-van Creveld syndrome also having an atrio-ventricular canal defect has been seen by two of us (HNN and DG). Thus it appears from this review that the developmental anomaly is directed towards the endocardial cushion and the final cardiac lesion is some form of common atrio-ventricular canal. This is strongly supported by the facts referred to in the previous paragraph and thus it appears that the identical cardiac lesion in both of our twins is not coincidental but genetic in origin.

The frequency of ectodermal dysplasia is 70 per cent but is not essential to the diagnosis. Its manifestations include sparsity of the hair, dystrophy of the fingernails, malformations of the teeth, absent gingival sulcus, hare-lip, epi- and hypospadias, and mental retardation (Caffey, 1952; Darling et al., 1957; Mitchell and Waddell, 1958). In our patients, there were no manifestations of ectodermal dysplasia. It should be pointed out, however, that they were the only blond representatives in a large family with black hair.

The appearance of a four-finger line has been described mainly in mongols (Beckman, Gustavson, and Norring, 1962). The appearance of the four-finger line in our patients is probably a secondary manifestation of the abnormal shortness of the metacarpal bones. It is significant that in mongolism also there is an abnormality of metacarpal bones (Hefke, 1940), which suggests the possibility of a mechanism common to both disorders.

In some patients with Ellis-van Creveld syndrome the ectodermal manifestations may be entirely absent. In others the changes may be partial, affecting only the nails, or the nails and teeth together, while the hair may be normal. In effect, the ectodermal component, just as the cardiac component, may be absent in some cases and this does not negate the diagnosis.

Partial forms of the syndrome with some cases referred to as forme fruste (Debre et al., 1955) have been described. In their cases there were all types of combinations of the features described. In our patients, 3 of the 4 major signs of the syndrome were present-chondrodystrophy, polydactyly, and a heart defect. Another interesting characteristic is the special sense of humour the patients possess, a feature that has also been observed by van Creveld.

\section{SUMMARY}

Identical twins, 17 years old, with the Ellis-van Creveld syndrome (forme fruste) identical in both of them are described. The chondrodystrophy was only discovered at the age of 12 . They had post-axial polydactyly of hands and feet and a heart defect of the persistent common atrio-ventricular canal type. The only ectodermal abnormalities that were found related to the four-finger line of the palms, as was discussed, and there were minor changes in the eyes. 
Although the descriptions of the heart lesion in reported cases are not always full, it appears that the most usual cardiac lesion is some form of common atrio-ventricular canal.

It seems that forme fruste of Ellis-van Creveld should be suspected in two situations. (1) When there is a family history of Ellis-van Creveld and one or more of the manifestations appear in the descendants. (2) When there is a combination of at least two of the following four lesions: (a) ectodermal dysplasia, (b) polydactyly, (c) chondrodystrophy, and (d) congenital heart lesion of the common atrio-ventricular canal type (single atrium or any partial or complete form of common atrio-ventricular canal).

We wish to express our thanks to Dr. A. Sternberg and Dr. A. Katzenelson for reviewing the X-ray pictures.

\section{REFERENCES}

Alvarez-Borja, A. (1960). Ellis-van Creveld syndrome. Pediatrics, 26, 301.

Beckman, L., Gustavson, K. H., and Norring, A. (1962). Finger and palm dermal tidges patterns in normal and mongoloid individuals. Acta Genet. (Basel), 12, 20.

Behar, A., and Rachmilewitz, E. (1964). Ellis-van Creveld syndrome. Arch. intern. Med., 113, 606.

Caffey, J. (1952). Chondroectodermal dysplasia (Ellis-van Creveld disease). Report of 3 cases. Amer. J. Roentgenol., 68, 875 .

Chauss, J. M. (1955). Chondroectodermal dysplasia (Ellis-van Creveld disease). Radiology, 65, 213.

Darling, R. C., Rothney, W. B., and Craig, J. M. (1957). Total pulmonary venous drainage into the right side of the heart. Lab. Invest., 6, 44.

Debré, R., Lamy, M., Minkowski, A., and Grumbach, E. (1955). Syndrome d'Ellis-van Creveld chez un nouveau-né décédé le premier jour. Arch. franç. Pédiat., 9, 1055.

Douglas, W. F., Schonholtz, G. J., and Geppert, J. L. (1959). Chondroectodermal dysplasia (Ellis-van Creveld syndrome). A.M.A. J. Dis. Child., 97, 473.

Ellis, R. W. B., and van Creveld, S. (1940). A syndrome characterized by ectodermal dysplasia, polydactyly, chondrodysplasia and congenital morbus cordis. Report of three cases. Arch. Dis. Childh., 15, 65.

-, and Andrew, J. D. (1962). Chondroectodermal dysplasia. J. Bone Jt Surg., 44-B, 626.

Ferrero, N. A., Pozo, O. O., and Morresi, E. S. (1961). Chondroectodermal dysplasia, J. Bone Jt Surg., 43-A, 1230.

Fontana, R. S., and Edwards, J. E. (1962). Congenital Cardiac Diseases: A Review of 357 Cases Studied Pathologically, p. 62. W. B. Saunders, Philadelphia and London.

Gatto, I. (1951). Ellis-van Creveld syndrome. Helv. padiat. Acta, 6, 437.

Giknis, F. L. (1963). Single atrium and the Ellis-van Creveld syndrome. J. Pediat., 62, 558.

Hefke, H. W. (1940). Roentgenologic study of anomalies of the hands in one hundred cases of mongolism. Amer. J. Dis. Child., 60, 1319.

Holt, L. E., and McIntosh, R. (1933). Holt's Diseases of Infancy and Childhood, 10th ed., p. 362. D. Appleton, New York.

Husson, G. S., and Parkman, P. (1961). Chondroectodermal dysplasia (Ellis-van Creveld syndrome) with a complex cardiac malformation. Pediatrics, 28, 285.

Keizer, D. P. R., and Schilder, J. H. (1951). Ectodermal dysplasia, achondrodysplasia and congenital morbus cordis. A.M.A. Amer. J. Dis. Child., 82, 341 .

Kushnick, T., Paya, K., and Mamunes, P. (1962). Chondroectodermal dysplasia. Amer. J. Dis. Child., 103, 77.

Lynch, R. J., and Engle, M. A. (1961). Familial congenital complete heart block (with polydactylism). Amer.J. Dis. Child., 102, 210.

MacGregor, M. (1954). Chondro-ectodermal dysplasia (Ellis-van Creveld syndrome) Colobomata of iris. Proc. roy. Soc. Med., 47, 540 .

McKusick, V. A. (1959). Genetic factors in cardiovascular diseases. I. The four major types of cardiovascular disease. Mod. Conc. cardiovasc. Dis., 28, 535.

Medulla, M. (1954). Ellis-van Creveld syndrome, or ectodermal chondrodysplasia. Pediat., Internat., 4, 1.

Metrakos, J. D., and Fraser, F. C. (1954). Evidence for a hereditary factor in chondroectodermal dysplasia (Ellis-van Creveld syndrome). Amer. J. hum. Genet., 6, 260.

Mitchell, F. N., and Waddell, W. W., Jr. (1958). Ellis-van Creveld syndrome: Report of two cases in siblings. Acta paediat. (Uppsala), 47, 142.

Neufeld, H. N., Titus, J. L., DuShane, J. W., Burchell, H. B., and Edwards, J. E. (1961). Isolated ventricular septal defect of the persistent common atrioventricular canal type. Circulation, 23, 685.

Ross, L. J. (1959). Congenital cardiovascular anomalies in twins. Circulation, 20, 327.

Ross, L. H. L., and Hand, A. M. (1958). Chondroectodermal dysplasia (Ellis-van Creveld syndrome). Report of
Smith, H. two cases. Pediatrics, 21, 298.

Toscano-Barbosa, E., Brandenburg, R. O., and Burchell, H. B. (1956). Electrocardiographic studies of cases with intracardiac malformations of the atrio-ventricular canal. Proc. Mayo Clin., 31, 513.

Turner, E. K. (1956). The Ellis-Creveld syndrome: Report of a case. Med. J. Aust., 1, 366.

Uehlinger, E. (1957). Pathologische Anatomie der chondro-ektodermalen Dysplasie Ellis-van Creveld. Schweiz. Z. allg. Path., 30, 754.

Walls, W. L., Altman, D. H., and Winslow, O. P. (1959). Chondroectodermal dysplasia (Ellis-van Creveld syndrome). A.M.A. J. Dis. Child., 98, 242.

Weiss, H., and Crosett, A. D. (1955). Chondroectodermal dysplasia. J. Pediat., 46, 268.

Weller, S. D. V. (1951). Chondroectodermal dysplasia (Ellis-van Creveld syndrome). Proc. roy. Soc. Med., $44,731$. 